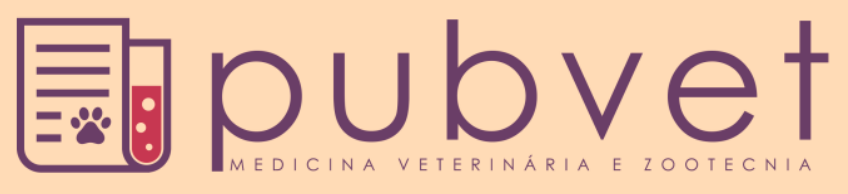

HTTP://DX.DOI.ORG/10.22256/PUBVET.V11N12.1293-1297

\title{
Dinâmica da leishmaniose visceral humana no município de Goiana - PE
}

\author{
Jonatas Abinadabe Oliveira Silva ${ }^{1 *}$, Gílcia Aparecida de Carvalho², Maria Aparecida da \\ Gloria Faustino ${ }^{3}$
}

${ }^{1}$ Veterinário formado pela Universidade Federal Rural de Pernambuco, Recife, PE, Brasil.E-mail: jonatas.vet@hotmail.com ${ }^{2}$ Professora da Universidade Federal Rural de Pernambuco, Unidade Acadêmica de Garanhuns, Garanhuns, PE, Brasil. Email: gilciasilva@yahoo.com.br

${ }^{3}$ Professora da Universidade Federal Rural de Pernambuco, Departamento de Doenças Parasitárias dos Animais Domésticos, Recife, PE, Brasil. E-mail: magfaustino@hotmail.com

*Autor para correspondência

RESUMO. A leishmaniose visceral (LV) encontra-se amplamente distribuída no território pernambucano, embora dados sobre a dinâmica e características epidemiológicas da doença em algumas áreas sejam ainda fragmentados e incipientes; esse é o caso do município de Goiana, onde casos de leishmaniose visceral em cães e em humanos têm sido registrados. O presente estudo tem como objetivo analisar o comportamento da doença no município de Goiana - PE no período de 2007 a 2015 através da utilização de indicadores epidemiológicos. Para isso, foram utilizados dados estatísticos referentes à notificação dos casos humanos de LV do Sistema Nacional de Agravos de Notificações e do Departamento de Informática do Sistema Único de Saúde do Brasil com o intuito de calcular indicadores epidemiológicos destinados a avaliação situacional da LV no município. Houve um total de 18 notificações de casos humanos confirmados para LV, incluindo 13 autóctones. Os cálculos dos coeficientes de incidência e prevalência confirmam a persistência e a intensidade da doença na população durante o período analisado. Foram 10 confirmações por exame laboratorial e 3 por parâmetros clínico-epidemiológicos. Com relação a evolução da doença, 10 pacientes tiveram a cura e não houve óbito por leishmaniose. Foi observado que $84,6 \%$ dos casos autóctones foram do gênero masculino. Apresentou uma maior frequência nos adultos entre 20 a 59 anos com 53,8\% do total de casos. No que diz respeito a zona de residência, $84,6 \%$ foram provenientes da zona rural. O município de Goiana apesar de não apresentar coeficientes epidemiológicos de caráter significante que denotem a ocorrência de uma epidemia, a presença de casos humanos autóctones ao longo do período estudado, deixam um alerta às autoridades governamentais e aos profissionais de saúde.

Palavras chave: Epidemiologia, leishmaniose visceral, zoonose

\section{Dynamics of human visceral leishmaniasis in the municipality of Goiana-Brazil}

ABSTRACT. The Visceral leishmaniasis (LV) is widely distributed in the territory of Pernambuco, although data on the dynamics and epidemiological characteristics of the disease in some areas are still fragmented and incipient; this is the case of the municipality of Goiana, where cases of visceral leishmaniasis in dogs and in humans have been recorded. The present study aims to analyze the behavior of the disease in the municipality of Goiana - PE in the period from 2007 to 2015 through the use of epidemiological indicators. For this, we used statistical data regarding the notification of human cases of LV of the National System of Notifiable Diseases and the Department of Informatics of the Brazil Unified 
Health System with the purpose of calculating epidemiological indicators intended for the situational assessment of LV in the municipality. There were a total of 18 reports of human cases confirmed for LV, including 13 autochthonous. The calculations of the incidence and prevalence coefficients confirm the persistence and intensity of the disease in the population during the analyzed period. There were 10 confirmations per laboratory tests and 3 per clinical-epidemiological parameters. With regard to the evolution of the disease, 10 patients were cured and there was no death by leishmaniasis. It was observed that $84.6 \%$ of the autochthonous cases were of the masculine gender. It presented a higher frequency in adults between 20 and 59 years of age with $53.8 \%$ of the total cases. As regards the area of residence, $84.6 \%$ came from the rural area. Although the municipality of Goiana does not present significant epidemiological coefficients that indicate the occurrence of an epidemic, the presence of autochthonous human cases during the studied period, leaves an alert to the governmental authorities and health professionals.

Keywords: Epidemiology, visceral leishmaniasis, zoonosis

\section{Dinámica de la leishmaniosis visceral humana en el municipio de Goiana-Brasil}

RESUMEN. La leishmaniosis visceral (LV) se encuentra ampliamente distribuida en el territorio pernambucano, aunque datos sobre la dinámica y características epidemiológicas de la enfermedad en algunas áreas sean todavía fragmentados e incipientes; como es el caso del municipio de Goiana, donde se han registrado casos de leishmaniosis visceral en perros y en humanos. El presente estudio tiene como objetivo analizar el comportamiento de la enfermedad en el municipio de Goiana - PE durante el período de 2007 a 2015 mediante la utilización de indicadores epidemiológicos. Se utilizaron datos estadísticos referentes a la notificación de los casos humanos de LV del Sistema Nacional de Agravios de Notificaciones y del Departamento de Informática del Sistema Único de Salud de Brasil con el propósito de calcular indicadores epidemiológicos destinados a la evaluación situacional de la LV en el municipio. Hubo un total de 18 notificaciones de casos humanos confirmados para LV, siendo 13 autóctonas. Los cálculos de los coeficientes de incidencia y prevalencia confirman la persistencia y la intensidad de la enfermedad en la población durante el período analizado. Fueron 10 confirmaciones por examen de laboratorio y 3 por parámetros clínico-epidemiológicos. En relación a la evolución de la enfermedad, 10 pacientes tuvieron la curación y no hubo muerte por leishmaniosis. Se observó que el 84,6\% de los casos autóctonos fueron del género masculino. Presentó una mayor frecuencia en los adultos entre 20 y 59 años con $53,8 \%$ del total de casos. Por lo que se refiere a la zona de residencia, el $84,6 \%$ procedían de la zona rural. El municipio de Goiana a pesar de no presentar coeficientes epidemiológicos de carácter significante que denoten la ocurrencia de una epidemia, la presencia de casos humanos autóctonos a lo largo del período estudiado, dejan una alerta a las autoridades gubernamentales y a los profesionales de la salud.

Palabras clave: Epidemiología, leishmaniosis visceral, zoonosis

\section{Introdução}

A leishmaniose visceral (LV) é uma zoonose crônica causada pelo protozoário Leishmania infantum (=Leishmania chagasi) cuja transmissão ocorre pelo repasto sanguíneo de uma fêmea de um flebotomíneo infectado (Almeida et al., 2010). Os vetores Lutzomyia longipalpis e Lutzomyia cruzi são as duas espécies de flebotómos que estão relacionadas com a transmissão da doença no Brasil. Em relação aos reservatórios, o cão é a principal fonte de infecção na área urbana; enquanto que no ambiente silvestre são considerados reservatórios as raposas e os marsupiais (Santos et al., 1998). No Brasil a LV é uma doença endêmica. No entanto têm sido registrados surtos frequentes que estão distribuídos em 21 unidades da federação, atingindo as cinco regiões brasileiras (Santos et al., 1998). 
Em Pernambuco, vários trabalhos descreveram a ocorrência da LV (Dantas-Torres and BrandãoFilho, 2006, Gontijo and Melo, 2004, Almeida et al., 2010, Silva and Braga, 2010). Segundo Almeida et al. (2010) a LV encontra-se amplamente distribuída no território pernambucano, embora dados sobre a dinâmica e características epidemiológicas da doença em algumas áreas sejam ainda fragmentados e incipientes; esse é o caso do município de Goiana, onde casos de leishmaniose visceral em cães e em humanos têm sido registrados.

Considerando que a incidência e a prevalência traduzem, respectivamente, a ideia de intensidade com a qual a morbidade incide sobre uma população em um intervalo de tempo e da força com que subsistem casos das doenças nas comunidades (Lima et al., 2014). Desta forma, justifica-se a realização do presente estudo com o intuito de construir estes indicadores epidemiológicos da LV do município de Goiana, além de outros dados.

O presente estudo tem como objetivo analisar o comportamento da LV em Goina no período de 2007 a 2015 pela utilização de indicadores epidemiológicos.

\section{Material e Métodos}

O estudo trata-se de uma análise documental e descritiva dos casos de LV no município de Goiana no período de 2007 a 2015. Este município está localizado no Estado de Pernambuco, região Nordeste do Brasil. O município está localizado nas coordenadas geográficas $07^{\circ} 33^{\prime} 38^{\prime \prime} \mathrm{S}$ (latitude) e $35^{\circ} 00^{\prime} 09^{\prime}$ 'W (longitude), a uma altitude de 13 metros (Base de dados do Estado, 2017).

Dados estatísticos referentes à notificação dos casos humanos de LV foram obtidos através do Sistema Nacional de Agravos de Notificações SINAN no período de 2007 a 2015. Os dados populacionais foram captados no site do Departamento de Informática do Sistema Único de Saúde do Brasil - DATASUS. Foram calculados indicadores epidemiológicos destinados à avaliação situacional da LV no município.

Também foram incluídos no estudo dados referente ao diagnóstico, evolução do caso, gênero, faixa etária e zona de residência.

\section{Resultados e discussão}

A série temporal em Goiana revela um total de
18 notificações de casos humanos confirmados para LV, sendo 13 autóctones. Dos casos autóctones, 10 foram registrados como novos.

A partir dos números de casos confirmados, coletados no site do SINAN foi calculado o coeficiente de prevalência e incidência dos casos autóctones (Tabelas 1 e 2 ). Para o período mencionado anteriormente, demonstrando como persiste a doença na região e a intensidade com a qual a morbidade incide sobre uma população em um intervalo de tempo.

Tabela 1. Coeficiente de prevalência da leishmaniose visceral no município de Goiana de 2007 a 2015.

\begin{tabular}{lcccc}
\hline Ano & $\begin{array}{c}\text { Caso } \\
\text { confirmado } \\
\text { autóctone }\end{array}$ & \multicolumn{3}{c}{$\begin{array}{c}\text { Coeficiente } \\
\text { por 10 mil } \\
\text { hab. }\end{array}$} \\
\hline 2007 & 4 & 76.071 & 0,53 & 0,005 \\
2008 & 3 & 76.479 & 0,39 & 0,0039 \\
2009 & 1 & 76.880 & 0,13 & 0,0013 \\
2010 & 3 & 77.271 & 0,39 & 0,0039 \\
2011 & 0 & 77.651 & 0 & 0 \\
2012 & 1 & 77.592 & 0,13 & $0,0013 \%$ \\
2013 & 0 & 77.945 & 0 & 0 \\
2014 & 1 & 78.289 & 0,13 & $0,0013 \%$ \\
2015 & 0 & 78.623 & 0 & 0 \\
\hline
\end{tabular}

Fonte: Sistema de Informação de Agravos de Notificação SINAN 2007 a 2015. Observação: Dados de casos confirmados de 2015 sujeitos a revisão pelo Ministério da Saúde.

Tabela 2. Coeficiente de incidência da leishmaniose visceral no Município de Goiana de 2007 a 2015.

\begin{tabular}{lcccc}
\hline Ano & $\begin{array}{c}\text { Caso } \\
\text { confirmado } \\
\text { autóctone } \\
\text { novo }\end{array}$ & População & $\begin{array}{c}\text { Coeficiente } \\
\text { por } 10 \text { mil } \\
\text { hab. }\end{array}$ & $\begin{array}{c}\text { Coeficiente } \\
\text { em } \\
\text { porcentage } \\
\text { m }\end{array}$ \\
\hline 2007 & 4 & 76.071 & 0,53 & 0,0053 \\
2008 & 2 & 76.479 & 0,26 & 0,0026 \\
2009 & 1 & 76.880 & 0,13 & 0,0013 \\
2010 & 2 & 77.271 & 0,26 & 0,0026 \\
2011 & 0 & 77.651 & 0 & 0 \\
2012 & 1 & 77.592 & 0,13 & 0,0013 \\
2013 & 0 & 77.945 & 0 & 0 \\
2014 & 0 & 78.289 & 0 & 0 \\
2015 & 0 & 78.623 & 0 & 0 \\
\hline
\end{tabular}

Fonte: Sistema de Informação de Agravos de Notificação SINAN 2007 a 2015. Observação: Dados de casos confirmados novos de 2015 sujeitos a revisão pelo Ministério da Saúde.

Observou-se que os coeficientes de incidência foram menores que os da prevalência nos anos de 2008, 2010 e 2014 e através destes coeficientes apresentados nas tabelas, confirmando a 
persistência e a intensidade da doença na população durante o período analisado, mesmo ainda que se apresente uma baixa incidência. Diante do exposto, segundo o critério do Ministério da Saúde que considera a média anual de casos novos autóctones dos 3 últimos anos pode-se classificar Goiana como município endêmico com transmissão esporádica, levando em consideração os anos de 2012 a 2014 (pois os dados de 2015 ainda estão sujeitos a revisão). No que se refere à confirmação diagnóstica da LV, a mesma se dá pelo exame laboratorial e critério clínico-epidemiológico e está demonstrado na Tabela 3. Considerando o total de notificações de casos autóctones (13) durante o período estudado, verificou-se que houve 10 confirmações por exames laboratoriais e 03 por parâmetros clínicoepidemiológicos. Convém salientar que o diagnóstico da LV se baseia em exames imunológicos (tais como imuno-fluorescência indireta, testes rápidos imuno-cromatográficos e ensaio imuno-enzimático) e parasitológicos, sendo este último considerado de certeza pelo encontro das formas amastigotas do parasito em material biológico obtido preferencialmente da medula óssea do paciente.

Tabela 3. Número de casos confirmados segundo critério de confirmação de paciente com leishmaniose visceral no Município de Goiana de 2007 a 2015.

\begin{tabular}{lcr}
\hline Ano/Critério & Laboratorial & Clínico-epidemiológico \\
\hline 2007 & 2 & 2 \\
2008 & 3 & - \\
2009 & 1 & - \\
2010 & 3 & - \\
2011 & - & - \\
2012 & 1 & - \\
2013 & - & - \\
2014 & - & 1 \\
2015 & - & - \\
\hline
\end{tabular}

Fonte: Sistema de Informação de Agravos de Notificação SINAN 2007 a 2015. Observação: Dados de 2015 sujeitos a revisão pelo Ministério da Saúde.

Na tabela 4 foi demonstrado o número de casos confirmados autóctones segundo a evolução do paciente e durante o período analisado 10 pessoas obtiveram a cura, houve 1 transferência e em 2 situações tiveram informação como ignorada e/ou branco. Não houve óbito por LV, o que reflete a eficácia do tratamento aos portadores de LV. Porém, vale salientar que houve um óbito por outra causa no ano de 2014, no entanto, era de um caso importado de LV que foi notificado no município.
Em relação ao gênero, dos 13 casos autóctones de LV em Goiana, 84,6\% (11/13) foram do gênero masculino e $15,4 \%$ (02/13) do sexo feminino. Estudos apontam para uma maior susceptibilidade do gênero masculino a esta parasitose (Góes et al., 2012).

Tabela 4. Número de casos confirmados autóctones segundo evolução do paciente com leishmaniose visceral no Município de Goiana de 2007 a 2015.

\begin{tabular}{lccc}
\hline Ano/Evolução & Cura & Transferência & Ignorado/Branco \\
\hline 2007 & 3 & 1 & - \\
2008 & 3 & - & - \\
2009 & 1 & - & - \\
2010 & 2 & - & 1 \\
2011 & - & - & - \\
2012 & - & - & 1 \\
2013 & - & - & - \\
2014 & 1 & - & - \\
2015 & - & - & - \\
\hline
\end{tabular}

Fonte: Sistema de Informação de Agravos de Notificação SINAN 2007 a 2015. Observação: Dados de 2015 sujeitos a revisão pelo Ministério da Saúde.

A doença se apresentou com maior frequência nos adultos entre 20 a 59 anos com 53,8\% (07/13) do total de casos, seguido das crianças com até 09 anos de idade com $38,5 \%(05 / 13)$ e a faixa etária de 15 a 19 anos com 7,7\% (01/13). Vale salientar que a doença em crianças não teve uma maior frequência como afirmam alguns estudos, levando em consideração que as crianças têm uma maior susceptibilidade a infecção (Góes et al., 2012).

No que diz respeito a zona de residência, observou-se que dos 13 casos autóctones da doença, 84,6\% (11/13) foram provenientes da zona rural e apenas $15,4 \%$ (02/13) da zona urbana. Recentemente a LV vem se expandindo para áreas urbanas de médio e grande porte, porém, primariamente se caracterizava como zoonose de caráter iminentemente rural. A maior frequência de casos oriundos da zona rural em Goiana corrobora com esta segunda afirmação. Vale salientar que no estado de Pernambuco, observouse que a endemia rural foi substituída pela doença urbana em função das modificações ambientais, à ocupação desordenada do espaço geográfico e as precárias condições de vida da população (DantasTorres and Brandão-Filho, 2006).

\section{Conclusão}

O conhecimento da dinâmica da leishmaniose visceral em Goiana através de indicadores epidemiológicos, como a prevalência e a 
incidência, além de outros dados, tornam-se uma ferramenta fundamental para a adoção de medidas eficazes na prevenção e tratamento da população.

O município de Goiana apesar de não apresentar coeficientes epidemiológicos de caráter significante que denotem a ocorrência de uma epidemia de LV, a presença de casos humanos autóctones ao longo do período estudado deixa um alerta às autoridades governamentais e aos profissionais de saúde para a existência de uma prática educativa e preventiva, como também do exercício constante de uma vigilância em saúde mais eficiente.

Os resultados apresentados neste estudo, podem ser tomados como referência à estudos futuros, permitindo uma comparação com resultados de áreas cujos indicadores epidemiológicos sejam variados, contribuindo para uma melhor apreciação do Programa Brasileiro de Controle da LV que prevê: detecção e tratamento dos casos humanos, controle de vetores e controle de reservatórios.

\section{Referências Bibliográficas}

Almeida, A. B. P. F., Mendonça, A. J. \& Sousa, V. R. F. 2010. Prevalência e epidemiologia da leishmaniose visceral em cães e humanos, na cidade de Cuiabá, Mato Grosso, Brasil. Ciência Rural, 40, 1610-1615.

Dantas-Torres, F. \& Brandão-Filho, S. P. 2006. Expansão geográfica da leishmaniose visceral no Estado de Pernambuco. Revista da Sociedade Brasileira de Medicina Tropical, 39, 352-356.
Góes, M. A. O., Melo, C. M. \& Jeraldo, V. D. L. S. 2012. Série temporal da leishmaniose visceral em Aracaju, estado de Sergipe, Brasil (1999 a 2008): aspectos humanos e caninos. Revista Brasileira de Epidemiologia, 15, 298307.

Gontijo, C. M. F. \& Melo, M. N. 2004. Leishmaniose visceral no Brasil: quadro atual, desfios e perspectivas. Revista Brasileira de Epidemiologia, 7, 338-349.

Lima, M. A. M., Maia, J. S., Zanetti, K., Reginaldo, A. S. \& Braz, P. H. 2014. Comparação da sensibilidade do teste parasitológico em linfonodo, medula óssea e mucosa conjuntival para o diagnóstico de leishmaniose em cães. Acta Veterinaria Brasilica, 8, 274-276.

Santos, S. O., Arias, J., Rribeiro, A. A., Hoffmann, M. P., Freitas, R. A. \& Malacco, M. A. F. 1998. Incrimination of Lutzomyia cruzi as a vector of American visceral leishmaniasis. Medical and Veterinary Entomology, 12, 315-317.

Silva, O. A. \& Braga, G. M. S. 2010. Leishmaniose visceral canina no município de São Vicente Férrer, Estado de Pernambuco, Brasil. PUBVET, 4, 879-885.

\section{Aticle History: \\ Received 31 July 2017 \\ Accepted 22 August 2017 \\ Available on line 25 October 2017}

License information: This is an open-access article distributed under the terms of the Creative Commons Attribution License 4.0, which permits unrestricted use, distribution, and reproduction in any medium, provided the original work is properly cited. 